\title{
An Astrobiological Experiment to Explore the Habitability of Tidally Locked M-Dwarf Planets
}

\author{
Daniel Angerhausen ${ }^{1}$, Haley Sapers ${ }^{2}$, Eugenio Simoncini ${ }^{3}$, Stefanie \\ Lutz $^{4}$, Marcelo da Rosa Alexandre ${ }^{5}$ and Douglas Galante ${ }^{6}$ \\ ${ }^{1}$ Rensselaer Polytechnic Institute (RPI), 110 Eighth Street, Troy, NY USA 12180 \\ email: angerd@rpi.edu \\ ${ }^{2}$ Centre for Planetary Science and Exploration, University of Western Ontario, Canada \\ ${ }^{3}$ Centro de Astrobiologa, INTA-CSIC, Madrid, Spain \\ ${ }^{4}$ School of Earth \& Environment, University of Leeds, UK \\ ${ }^{5}$ Chemistry Department, Federal University of Sergipe, Brazil \\ ${ }^{6}$ Instituto de Astronomia, Geofísica e Ciências Atmosféricas, Universidade de São Paulo, Brazil
}

\begin{abstract}
We present a summary of a three-year academic research proposal drafted during the Sao Paulo Advanced School of Astrobiology (SPASA) to prepare for upcoming observations of tidally locked planets orbiting M-dwarf stars. The primary experimental goal of the suggested research is to expose extremophiles from analogue environments to a modified space simulation chamber reproducing the environmental parameters of a tidally locked planet in the habitable zone of a late-type star. Here we focus on a description of the astronomical analysis used to define the parameters for this climate simulation.
\end{abstract}

Keywords. Astrobiology, habitable zone, extremophiles, M-Dwarf planets, tidally locked

\section{Introduction}

Detection and characterization of extrasolar Earth-sized planets is challenging as they are particularly small in comparison to their bright and massive host stars. Around F, G, and $\mathrm{K}$ type host stars, the easiest planets to detect using occultation/transit or kinematic techniques lie relatively close to the parent star, hence are probably uninhabitable due to the higher flux of stellar radiation (Kasting et al., 1993). Terrestrial planets in the habitable zone of M-dwarf stars, however, are easier to detect because for example such systems yield deeper, more frequent, and more probable transits as well as stronger radial-velocity signatures. The long lifetime of late-type dwarfs improve the chances of life being present by, for example, allowing sufficient time for stable, potentially habitable conditions to arise as well as sufficient time for the evolution of life. Also, M-dwarfs comprise $80 \%$ of main sequence stars in the solar neighborhood and the latest Kepler results suggest that they are significantly more likely to host rocky and close-in planets (Howard et al., 2012), therefore offering the best statistical chance to find and characterize habitable extrasolar worlds.

Planets close enough to M-dwarf stars to be located within the habitable zone are likely to be tidally locked (Dole, 1964; Fig. 1, A). Here we propose to study the habitability of tidally locked planets, focusing on one particular possible habitable configuration for such a planet at the outer, colder edge of the habitable zone: an Eyeball Earth. An Eyeball Earth may exist where water is present in a large enough fraction to produce an icy shell with a liquid ocean at the substellar point (Pierrehumbert, 2011; Pierrehumbert \& 
Gaidos, 2011). As Eyeball Earths in this configuration maintains liquid water combined with relatively strong observable differences between their day and night side, they are potentially the easiest habitable terrestrial planets to detect and characterize using a combination of transit, eclipse and phase curve observations.

\section{Research plan}

According to our proposed research plan (Angerhausen et al., 2013) an astrophysics team will define the range of conditions around M-dwarf stars where planets can exist in eyeball states and based on these models, derive the most probable set of astronomical, geological and chemical parameters to describe the planet's climate (atmosphere, soil composition, stellar irradiation etc.). Using these results, in the second phase of our proposal, a biology team will determine the habitability potential for putative organisms in Eyeball Earth environments using a modified planetary/space simulation chamber.

\subsection{Astrophysical characterization}

\subsubsection{Stellar Irradiation}

Studies of X-ray, UV and radio emission from active low-mass stars show that a large amount of energy is released at non-optical wavelengths. However, the small sample sizes and shallow volumes limit our ability to understand the prevalence and duration of the non-optical radiation. With regard to the activity of M-dwarf stars, the main goal of the observational astronomy proposal is to close this knowledge gap by conducting: (i) optical and (near-)infrared spectroscopic observational surveys of M-dwarf stars to determine the spectral distribution of their continuous radiation as well as stellar parameters (ii) multiband time-series observations of M-dwarfs to determine the frequency, intensity and spectral distribution of stellar activity. Special focus here will be in wavelength regimes with implications for habitability, such as the 250-300 nm absorption bands of protein and amino acids (Coulter et al., 1936).

The MUSCLES survey (Measurements of the Ultraviolet Spectral Characteristics of Low-mass Exoplanetary Systems, France et al., 2012) is a good example for the kind

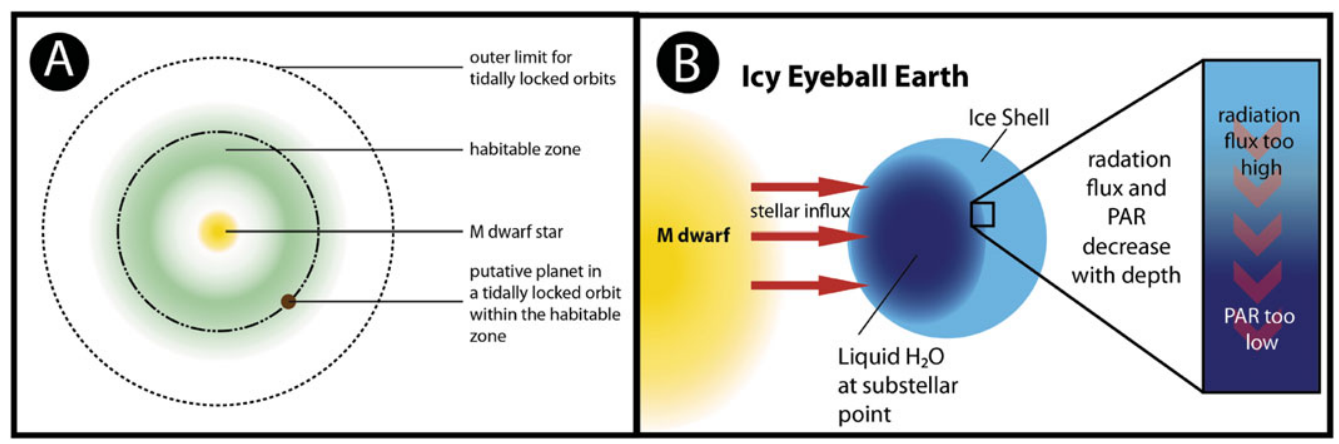

Figure 1. A: Putative M-Dwarf planetary system. Planets orbiting within the habitable zone surrounding an M-dwarf star will most likely be tidally locked. B: An icy Eyeball Earth orbiting at the right distance may have a liquid water ocean at the substellar point surrounded by an icy shell. The interface between the ice shell and the liquid water ocean may be potentially habitable. Characterization experiments conducted with analogue microbial communities from the Antarctic ice shelf will for example determine the optimal distance within the water column that will provide adequate protection from stellar radiation yet allow enough PAR through the ice layer to allow for photosynthetic metabolism and temperatures consistent with metabolically active life as we know it on Earth 
of observations needed in this study to define the simulated irradiation levels for the planetary chamber.

\subsubsection{Atmospheric Thermo- and Photochemistry}

We propose to develop a set of models for atmospheric chemistry and potential atmospheric, liquid and solid water, and other solid surface interaction processes. These models will be based on a set of variable assumptions of the main characteristics of the planet (temperature, pressure, incoming irradiation, possible composition of primitive atmosphere, outgassing products and main geology). In order to provide correct models and to use time-dependent parameters, backward kinetics will be included and computed by considering the actual, non-standard conditions (Simoncini \& Delgado-Bonal, 2013).

Due to the possible complexity of the atmosphere of an Eyeball Earth, thermo- and photochemical models need to be developed within a Global Circulation Model (GCM) (Heng \& Vogt, 2011). Such modeling will provide understanding about the day/night variations of thermodynamic conditions and, moreover, about the coupling between atmospheric convection processes and chemical kinetics. The chemical models will be analyzed from a thermodynamic point of view: when the concentration of reactants is different from estimations at equilibrium, one or more free energy inputs must be present: energy and/or material inflows into the atmosphere (abiotic or biotic); fast vertical mixing; and/or photochemistry. We propose the use of chemical disequilibrium to measure the free energy available to maintain life (both by atmospheric and gas/water/minerals redox processes) in addition to assessing possible signature of life based on the planet thermodynamic state (Lovelock, 1975). The disequilibrium associated with the chemical composition of the atmosphere will be computed by calculating the entropy production of each reaction (Kondepudi \& Prigogine, 1996).

This methodology provides a quantitative assessment of disequilibrium from different reactions and processes. Such chemical disequilibrium analyses will enable us to characterize the available energy and assess the thermodynamic implications for microbial metabolism.

As chemical disequilibrium is not dependent on a particular molecule but on the complex global condition, such analysis will provide a more objective and complete evaluation of the habitability of the considered planet (Lovelock, 1975).

\subsection{Biological analysis}

The biological objectives of our academic proposal is to determine the potential habitability of an Eyeball Earth by carrying out simulation experiments with a genetically characterized microbial community from a terrestrial analogue environment in modified a planetary simulation chamber reflecting the modelledmodeled parameters for an icy Eyeball Earth. Concomitantly with the astronomical characterization, we propose to conduct metagenomic and functional gene analysis of microbial communities thriving in Earth analogue environments, followed by determination of optimal habitability depth (Fig. 1B).

Firstly, the habitability potential of an Eyeball Earth environment needs to be determined. Identifying possible active microbial metabolisms will help us to identify detectable biosignatures produced on an Earth-like exoplanet which could be remotely sensed. Metabolisms driven by photosynthesis close to the surface as well as chemolitotrophy in the subsurface may be possible. Since conditions at the surface would likely be very harsh due to strong radiation, a putative photosynthetic community might survive and thrive deeper in the surface where photosynthetic active radiation (PAR) is still available and the microbial community is protected from harming radiation. Based on our 
astronomical models and observations PAR depth limits can be established and further examined by conducting field analysis on the ice in Antarctica. Photosynthetic organisms are limited by thermostability of chlorophyll which is not stable at temperatures exceeding 70C. Light-independent communities could be supported by active submarine hydrothermal systems, assuming a geologically active planet. These chemolithoautotrophic communities might not only be found in the deep lithosphere but closer to the surface shielded from harmful radiation.

The main goal will be to combine all calculations and analysis and adjust an existing simulation chamber (Brazilian Astrobiology Laboratory (Astrolab)) to modeled parameters reflecting icy Eyeball Earth conditions and conduct simulation experiments with UV and cold resistant microbial species. Microbial sampling will be conducted in Antarctica at the water-ice interface. Metagenomic and functional gene analysis of the microbial communities will be carried out yielding information about the dominant species and metabolisms in this environment. Identifying possible active microbial metabolisms will lead to the indentification of potential biosignatures produced on an Earth-like exoplanet which could be remotely detected with next generation (post-JWST) astronomical platforms.

\subsection{Experimental simulations}

In addition to the theoretical modeling of the conditions on tidally locked M-Dwarf planets, the research plan includes the development of techniques to produce experimental simulations of tidally locked planets. This approach opens the possibility of combining the knowledge acquired on the different phases of the project (astronomical observations, biological prospection of extreme environments on Earth and theoretical modeling of the exoplanets), in order to understand how real biological systems would behave in an extraterrestrial scenario. Two approaches will be followed in the simulation: (i) prebiotic scenarios, as interface and out of equilibrium conditions may be essential for the molecular complexification and eventual origin of life; (ii) survival and biological response of terrestrial extremophiles, that may produce potentially detectable biosignatures

While the astrophysical team works on the definition of the simulation parameters, the main focus will be on recreating these dynamical conditions expected to be found on the interface between the ice and liquid-water ocean of the planet. This project is based on the expansion of the capabilities of the already existent simulation chamber of the Astrobiology Laboratory at the University of Sao Paulo, Brazil. In a preliminary analysis, the chamber should be able to simultaneously simulate many parameters: temperature (tens to a few hundreds of K), ice-liquid transitions under a variety of pressures, pressure (vacuum to low pressures), gas composition (capacity to dynamically vary and monitor the gas composition inside the chamber), radiation (the chamber should be able to accommodate as many radiation sources as possible, such as UV lamps and ion guns, but it should be made ready to be used in nuclear and synchrotron accelerators, to expand the energies and fluxes available, so that the influence of stellar flares can be studied). As the interface of the planet is the main focus of the research, the chamber should be able to create and sustain dynamical and out of equilibrium conditions, including periodic and non-periodic variations of all parameters. This capacity is necessary to study oscillatory and transient phenomena on the system.

\section{Summary}

Extrasolar planets orbiting within the habitable zone of M-dwarf host stars are expected to play a significant role in the discovery of habitable environments beyond Earth. 
The habitable zone of dwarf stars is located relatively close to the host star, such that exoplanets orbiting within this zone will likely be tidally locked. On terrestrial planets with an icy shell, this may produce a liquid water ocean at the substellar point. Here we propose to conduct astronomical and geochemical research in order to define parameters needed to simulate potentially habitable environments on an icy Eyeball Earth planet. Using the interdisciplinary results of both the physical and biological teams we propose to set up a simulation chamber to expose a Earth analogue cold- and UV-tolerant microbial community to the theoretically derived climate states, simulating the composition, atmosphere, physical parameters and stellar irradiation. The results of the proposed study will enable us to derive observable parameters and potential biomarkers as well as target decision guidance and sensitivity requirements for the next generation of astronomical platforms. This interdisciplinary effort will require the contribution of students and researchers of different specialties, and represents well the challenges posed by scientific questions in astrobiology, which can help us advance our understanding of life in the Universe.

\section{References}

Kasting, J. F., Whitmire, D. P., \& Reynolds, R. T. 1993, Icarus, 101, 108

Howard, A. W., Marcy, G. W., Bryson, S. T., et al. 2012, ApJs, 201, 15

Dole, S. H. 1964, New York, Blaisdell Pub. Co. 1st ed.

Pierrehumbert, R. T. 2011, ApJl, 726, L8

Pierrehumbert, R. \& Gaidos, E. 2011, ApJl, 734, L13

Angerhausen, D., Sapers, H., Citron, R., et al. 2013, Astrobiology, 13, 309

Coulter, C. B., Stone, F. M., \& Kabat, E. A., 1936, JGP, vol. 19, no. 5, 739-752

France, K., Tian, F., Linsky, J. L., et al. 2012, AAS Meeting Abstracts, 220, 129.07

Simoncini, E. \& Delgado-Bonal, A., 2014, submitted

Heng, K. \& Vogt, S. S., 2011, MNRAS, 415, 2145

Kondepudi, D. \& Prigogine, I., 1996, Modern Thermodynamics (Wiley)

Lovelock, J. E., 1975, Proc. R. Soc. Lond., B. 189, 167 